\title{
Adult Guardianship in Korea: What Do Psychiatrists Need to Know?
}

\author{
Hyun Kook Lim $\bowtie$ \\ Department of Psychiatry, Yeouido St. Mary's Hospital, College of Medicine, The Catholic University of Korea, Seoul, Republic of Korea
}

Patients with mental illness such as schizophrenia, bipolar disorder, developmental disorder, and Alzheimer's disease lack decision making capacity; an ability of people to make decisions about utilizing information for their daily lives and decisions that are congruent with their values and preferences. ${ }^{1}$ Previously, incompetent or quasi-incompetent persons were registered with the family relationship registry, and they were prone to lose their all legal rights including rights to handle their personal welfares and financial affairs. Thus, the patients needing guardianship, called ward, or their family members used to avoid such registration due to social stigma. To protect human rights of the incompetent persons, the new Adult Guardianship Act 2011, aimed at enhancing right of self-determination by respecting an individual's residual capacity, was implemented in July 2013. ${ }^{2}$ The new adult guardianship is designed to help decision capacity of the wards in the areas including property management, physical wellbeing, medical care, and other personal welfares. Furthermore, a person without mental problem may also benefit from using the adult guardianship program by preparing for incompetence which may occur to them in the future. Since elderly populations are growing faster than any other age group across the world, the adult guardianship might help the elderlies with various mental and physical problems, such as Alzheimer's disease, to take better care of themselves and make proper decisions for their benefits.

There are three types of adult guardianship under the reformed Adult Guardianship Act in South Korea. ${ }^{2}$ In full guardianship, the adult guardians are responsible of the ward's medi$\mathrm{cal}$, residential, and other wide range of decisions regarding personal care. An adult guardian is appointed by the court for

\footnotetext{
$\triangle$ Correspondence: Hyun Kook Lim, MD, PhD

Department of Psychiatry, Yeouido St. Mary's Hospital, College of Medicine, The Catholic University of Korea, 10 63-ro, Yeongdeungpo-gu, Seoul 07345, Republic of Korea

Tel: +82-2-3779-1048, Fax: +82-2-780-6577, E-mail: drblues@catholic.ac.kr

(c) This is an Open Access article distributed under the terms of the Creative Commons Attribution Non-Commercial License (https://creativecommons.org/licenses/bync/4.0) which permits unrestricted non-commercial use, distribution, and reproduction in any medium, provided the original work is properly cited.
}

an adult who continuously lacks the capacity to conduct his/ her own job owing to mental constraint out of disease, disability, old age, and other reasons. Limited guardianship involves the guardians with specific responsibilities, such as health care and housing, with the ward retaining all other decisionmaking authorities. Specified guardianship may be granted for a limited period in response to an emergency situation, while the time-consuming process of obtaining full guardianship is under the process.

In these regards, the adult guardianship is a crucial legal policy that allows a person or an entity to make proper decisions for the incompetent or the ward. Considering its importance and significant personal as well as social impact, few issues around the adult guardianship should be taken into consideration. First, there was considerable variability in assessment of decision making capacity of the ward. According to the civil law, evidence of impaired decision-making capacity is a legal requirement for adult guardianship. Decision making capacity refers to the ability to make the judgment, as to whether an individual has the requisite cognitive, decisional, affective, and practical skills to adequately complete a particular task or make a particular decision. ${ }^{1,3}$ However, no consistent assessment method investigating decision making capacity exit, which may hinder the proper objective judgment for applications of 3 types of adult guardianship. Therefore, consensus among the experts and development of objective decision making capacity assessment tools are needed in the future. Second, psychiatrists need to have a better knowledge about legal impact of their psychiatric evaluation on the ward. Indeed, if the ward is under control of the adult guardianship, his/her legal rights on managing properties are considerably limited by the adult guardian. Hence, the psychiatrist's better understanding of these legal impact will enhance more accurate, thorough assessments of the decision making capacities of the ward receiving adult guardianship.

In conclusion, the psychiatric evaluation assessing the decision making capacities of the ward by a psychiatrist is a critical court proceedings for the adult guardianship. Therefore, the 
psychiatrists must be fully aware of the legal consequences of their clinical decision and should provide objective and reasonable evaluation results. In order to so, the psychiatrists should assess decision making capacity of the ward using multidimensional approach by taking the biological, psychological, and social obstacles inhibiting the incompetent's health, safety, and access to proper care in the community in to considerations.

\section{REFERENCES}

1. Moye J, Marson DC. Assessment of decision-making capacity in older adults: an emerging area of practice and research. J Gerontol B Psychol Sci Soc Sci 2007;62:p3-p11.

2. Ministry of Justice of Korea. The English translation of the Civil Act. Republic of Korea; 2016.

3. McSwiggan S, Meares S, Porter M. Decision-making capacity evaluation in adult guardianship: a systematic review. Int Psychogeriatr 2016;28: 373-384. 